\title{
Emittance control in rf cavities and solenoids
}

\author{
Mohammad Eshraqi, ${ }^{1, *}$ Giuliano Franchetti, ${ }^{2}$ and Alessandra M. Lombardi ${ }^{3}$ \\ ${ }^{1}$ CERN, CH-1211 Geneva 23, Switzerland, \\ and School of Accelerators and Particles, IPM (Institute for Research in Fundamental Sciences), P.O. Box 19395-5531, Tehran, Iran \\ ${ }^{2}$ GSI Darmstadt, D-64291 Darmstadt, Germany \\ ${ }^{3}$ CERN, CH-1211 Geneva 23, Switzerland \\ (Received 20 May 2008; published 25 February 2009)
}

\begin{abstract}
We study emittance growth for transport of uniform and Gaussian beams of particles in rf cavities and solenoids and show analytically its dependence on initial beam parameters. Analytical results are confirmed with simulation studies over a broad range of different initial beams.
\end{abstract}

DOI: 10.1103/PhysRevSTAB.12.024201

PACS numbers: 29.20.Ej, 41.85.- p, 84.32.Hh

\section{INTRODUCTION}

One of the challenges in the design of modern high power ion LINACs is the control of transverse emittance growth [1-3]. The transverse emittance is formed in the ion source when the beam is extracted out of plasma. Keeping the emittance growth to a minimum during the subsequent manipulations (longitudinal capture and matching) is of very high importance not only to deliver a high quality beam to the users, but also to limit the activation and the cost of the LINAC itself. An uncontrolled emittance growth and halo formation requires one to design the machine with a generous ratio between the bore aperture and the beam size and to design the shielding for heavy beam losses. This approach, i.e., to overdesign, leads to high costs and to heavy irradiation of the accelerator components which in turn excludes hands-on maintenance. Under the influence of purely linear forces emittance is conserved. The emittance growth generated by the beam self-forces and by mismatch have already been thoroughly investigated and recipes to control emittance growth are commonly applied in LINACs from the design stage [4,5]. Nevertheless there are still mechanisms causing emittance growth in the LINACs low-energy end that are revealed when a magnetic element or a rf cavity is represented with a field map instead of a lumped element or a finite element with hard-edge approximation. In this paper we concentrate on the study of emittance growth in solenoids and $\mathrm{rf}$ cavities, typically used for matching from the source to the first stage of rf acceleration and for adapting the bunch-tobucket injection between two stages of accelerations. Our reference layout is LINAC4 $[6,7]$ a new $\mathrm{H}^{-}$injector under study at CERN.

\section{A. Reference layout}

The layout of LINAC4 is sketched in Fig. 1. It consists of a rf volume source (identical to the one in DESY [8]) which provides an $\mathrm{H}^{-}$beam at $35 \mathrm{kV}$ further postaccel-

*meshraqi@cern.ch erated to $95 \mathrm{keV}$. The first rf acceleration (from $95 \mathrm{keV}$ to $3 \mathrm{MeV}$ ) is done by a radio frequency quadruple (the IPHI RFQ from CEA [9]). The RFQ resonates at $352 \mathrm{MHz}$, and is $6 \mathrm{~m}$ long. At $3 \mathrm{MeV}$ the beam enters a 3.6 meter long chopper line, consisting of 11 quadruples, three bunchers, and two sets of deflecting plates. As described in [6], the beam pulse length is $400 \mu \mathrm{s}(\max$. rep. rate $=2 \mathrm{~Hz}$ ), source current during the pulse is $80 \mathrm{~mA}$ and the chopper output current is $65 \mathrm{~mA}$. This system has the capability of removing microbunches on the rf scale (assumed chopping scheme is $222 / 133$ full to empty buckets) and rematch the beam to the subsequent system of accelerators. This reduces the LINAC average current to about $40 \mathrm{~mA}$, although for space-charge considerations, it is still a $65 \mathrm{~mA}$ beam. A rudimentary collimation is also performed in this line. The beam is then further accelerated to $40 \mathrm{MeV}$ in a conventional drift tube LINAC (DTL) at $352 \mathrm{MHz}$. The DTL, subdivided in three tanks, is 13.4 meters long. Each of the 82 drift tubes is equipped with a permanent magnet quadruple. At $40 \mathrm{MeV}$ the velocity of the beam is such as to allow the transition to structures which do not follow cell-by-cell the beam velocity profile. In LINAC4 the acceleration from 40 to $90 \mathrm{MeV}$ is provided by a cellcoupled drift tube LINAC (CCDTL) at $352 \mathrm{MHz}$. The CCDTL is made of 24 tanks of three cells each for a total length of 25.3 meters. The focusing is provided by electromagnetic quadruples placed outside each tank. The acceleration from 90 to $160 \mathrm{MeV}$ is done in a side coupled LINAC (SCL) resonating at $704 \mathrm{MHz}$. The SCL is made of 20 tanks of 11 cells each for a total of $28 \mathrm{~m}$. Focusing is provided by 20 electromagnetic quadruples [6]. This brings the total length of the LINAC to $80 \mathrm{~m}$, for a total of 18 klystrons, 55 electromagnetic quadruples, and 82 permanent magnet quadruples.

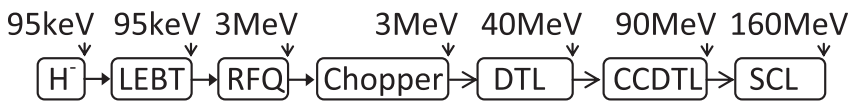

FIG. 1. Layout of LINAC4, not to scale. 


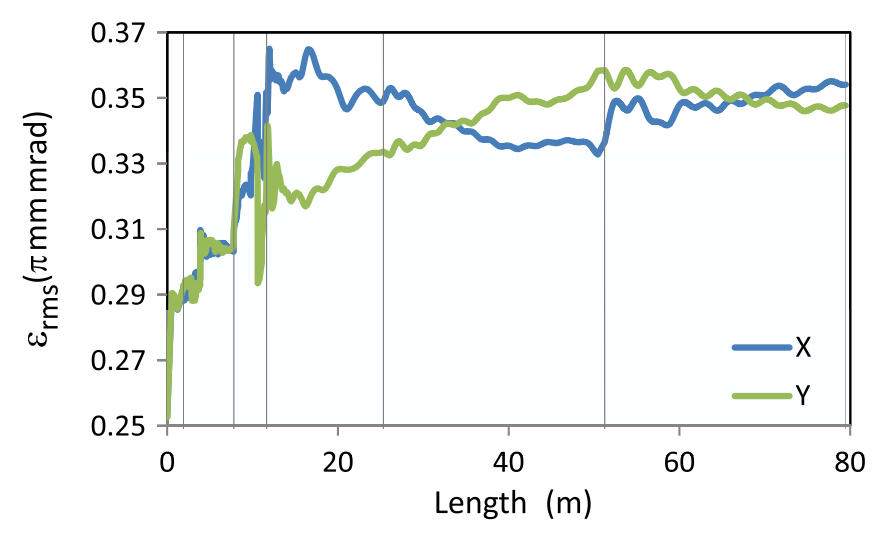

FIG. 2. (Color) Transverse emittance evolution along LINAC4, different sections which are separated with lines are LEBT, RFQ, Chopper, DTL, CCDTL, and SCL, respectively.

The end-to-end beam dynamics for the nominal beam and nominal LINAC, i.e., for a matched beam of $65 \mathrm{~mA}$ and no machine errors has been reported in [10] and it shows that the overall emittance growth is $40 \%$. The emittance growth is localized in the first solenoid of the low-energy beam transport (LEBT), in the coupling gap of
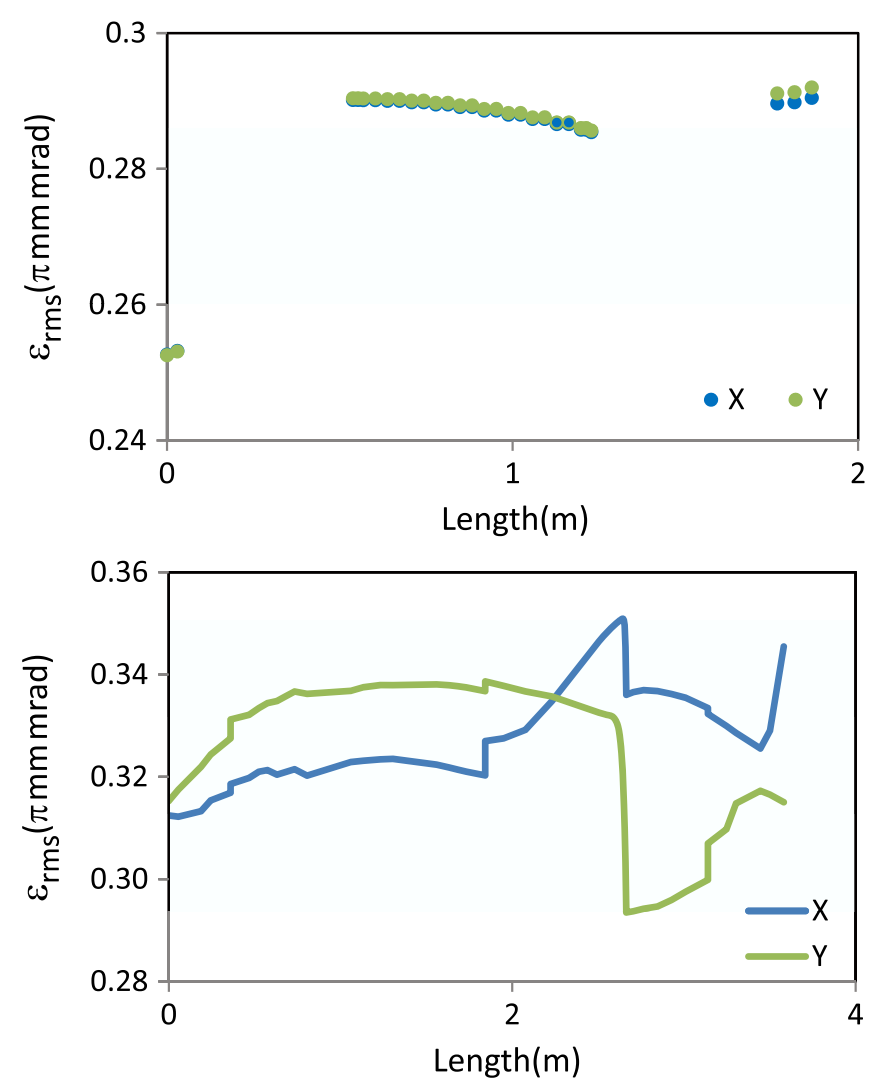

FIG. 3. (Color) Transverse emittance evolution. Top: LEBT with $8 \mathrm{~mA}$, including $90 \%$ space-charge neutralization in the LEBT, the emittance inside the solenoid is not indicated (out of scale). Bottom: Chopper line with $65 \mathrm{~mA}$. Positions of the rf cavities are marked with a vertical line. the RFQ, and in the buncher cavities of the chopper line. The plot of the emittance evolution all over the LINAC is shown in Fig. 2. A zoom of the LEBT and chopper line is shown in Fig. 3. The significant growth of the transverse emittance in rf cavities and solenoids motivated us to look for the optimal beam conditions at the entrance of a cavity or a solenoid in order to limit emittance growth and beam degradation.

\section{ANALYTICAL ESTIMATE OF EMITTANCE GROWTH}

In this section we attempt to derive a relation between the emittance variation when a beam passes through a solenoid or a rf cavity and the beam size and divergence when the beam passes through an electric field of a cavity or magnetic field of a solenoid. These relations will be verified numerically in the next section.

\section{A. Figures of merit}

We have chosen as a figure of merit the rms emittance, $\epsilon_{\mathrm{rms}}$, and the halo parameter, $H$. The variation of the emittance gives us a measure of the overall beam quality degradation whereas the halo parameter gives us a measure of the halo, potential losses, and consequent activation of the machine, as particles in the halo are the ones most likely to be lost under the effect of machine imperfection or beam misalignment. For completeness their definition is reported below.

The term rms emittance, $\epsilon_{\mathrm{rms}}$, refers to the area of an ellipse of the rms beam projection in the phase space plane. For an upright ellipse this value is equal to $\pi p_{i} q_{i}$, where $p_{i}$ and $q_{i}$ are canonically conjugate variables representing position and momentum in the $i^{\text {th }}$ plane. To avoid the ambiguity caused by different definitions of emittance, the value $\pi$ is quoted in the units. For a more general case which includes tilted ellipses as well as upright ones, the general expression reads

$$
\epsilon_{i_{\mathrm{rms}}} \equiv \pi \sqrt{\left\langle q_{i}^{2}\right\rangle\left\langle p_{i}^{2}\right\rangle-\left\langle q_{i} p_{i}\right\rangle^{2}}
$$

The halo in each plane $i$, for continuous, and bunched beams is defined [11] introducing the two invariants of motion,

$$
\begin{aligned}
I_{2}^{i} & \equiv\left\langle q_{i}^{2}\right\rangle\left\langle p_{i}^{2}\right\rangle-\left\langle q_{i} p_{i}\right\rangle^{2}, \\
I_{4}^{i} & \equiv\left\langle q_{i}^{4}\right\rangle\left\langle p_{i}^{4}\right\rangle+3\left\langle q_{i}^{2} p_{i}^{2}\right\rangle^{2}-4\left\langle q_{i}^{3} p_{i}\right\rangle\left\langle q_{i} p_{i}^{3}\right\rangle .
\end{aligned}
$$

The halo parameter $H$ in the $i^{\text {th }}$ plane is defined as

$$
H_{i}^{c} \equiv \frac{\sqrt{3 I_{4}^{i}}}{2 I_{2}^{i}}-2,
$$

and for bunched beam of particles is defined as 


$$
H_{i}^{b} \equiv \frac{\sqrt{3 I_{4}^{i}}}{2 I_{2}^{i}}-\frac{15}{7} .
$$

Normalization factors are defined such that the halo parameter for a continuous beam is equal to 0 for a uniform distribution and 1 for a Gaussian distribution.

\section{B. rf cavities}

The electric field and induced magnetic field in a pillbox cavity excited in the $T M_{010}$ mode can be written as [12]

$$
\begin{aligned}
& E_{z}(\rho, z, t)=\sum_{m} \mathrm{~A}_{m} I_{0}\left(k_{m} \rho\right) \cos \left(\frac{\pi m z}{L}\right) \sin \omega t, \\
& E_{\rho}(\rho, z, t)=\sum_{m} \frac{\mathrm{A}_{m} m \pi}{k_{m} L} I_{1}\left(k_{m} \rho\right) \sin \left(\frac{\pi m z}{L}\right) \sin \omega t, \\
& B_{\theta}(\rho, z, t)=\sum_{m} \frac{\mathrm{A}_{m} \omega}{k_{m} c^{2}} I_{1}\left(k_{m} \rho\right) \cos \left(\frac{\pi m z}{L}\right) \cos \omega t,
\end{aligned}
$$

where $\rho$ stands for the radial distance from design orbit, $z$ varies along the design orbit, $I_{m}$ are modified Bessel functions, $I_{m}(x)=i^{-m} J_{m}(i x)$, where $i=\sqrt{-1}, L$ is the field length, $k_{m}^{2}=(m \pi / L)^{2}-(2 \pi / \lambda)^{2}, \lambda$ is the wavelength, $t$ is the time, and $\omega=2 \pi c / \lambda$ is the angular frequency.

By using the expansion of Bessel function [13],

$$
J_{m}(x)=\sum_{j} \frac{(-1)^{j}}{\Gamma(j+1) \Gamma(j+m+1)}\left(\frac{x}{2}\right)^{2 j+m},
$$

and keeping up to the third power of radius,

$$
\begin{aligned}
E_{z}(\rho, z, t)= & E_{0} \sum_{m} A_{m}\left[1+\left(\frac{k_{m} \rho}{2}\right)^{2}\right] \cos \left(\frac{\pi m z}{L}\right) \sin \omega t, \\
E_{\rho}(\rho, z, t)= & E_{0} \sum_{m} \frac{A_{m} m \pi}{2 k_{m} L}\left[k_{m} \rho+\left(\frac{k_{m} \rho}{2}\right)^{3}\right] \\
& \times \sin \left(\frac{\pi m z}{L}\right) \sin \omega t, \\
B_{\theta}(\rho, z, t)= & E_{0} \sum_{m} \frac{A_{m} \omega}{2 k_{m} c^{2}}\left[k_{m} \rho+\left(\frac{k_{m} \rho}{2}\right)^{3}\right] \\
& \times \cos \left(\frac{\pi m z}{L}\right) \cos \omega t,
\end{aligned}
$$

while

$$
E_{0}=\frac{1}{L} \int_{-L / 2}^{L / 2} E_{z}(0, z) d z
$$

where $E_{z}(0, z)$ is the longitudinal electric field on axis.

The impulse, change in the momentum, due to the electric radial field and magnetic azimuthal field to the beam is

$$
\begin{aligned}
\Delta p_{\rho} & =\int F d t=\int F \frac{d s}{v}=\frac{1}{\beta c} \int F d s \Rightarrow \Delta p_{\rho} \\
& =\frac{q \Delta s}{\beta c}\left(E_{\rho}-\frac{\beta}{c} B_{\theta}\right),
\end{aligned}
$$

where $p_{\rho}$ is the radial momentum, since there is no azimuthal force on the particle hereafter it will be denoted by $p_{x}$, $F$ is the radial force, $q$ is the electric charge of the particle, $\Delta s$ is the length of the field increment, and $\beta$ is the average reduced velocity. The divergence of a particle having a radial distance of $\rho$ from design axis after passing through the cavity will be

$$
x_{f}^{\prime}=x_{i}^{\prime}+\Delta x^{\prime},
$$

where subscripts $i$ and $f$ refer to initial and final values, and

$$
\begin{aligned}
\Delta x^{\prime}= & \frac{\Delta p_{x}}{p_{z}} \\
= & \frac{q E_{0} \Delta s}{2 p_{z} \beta c} \sum_{m} \frac{A_{m}}{k_{m}}\left[k_{m} x+\left(\frac{k_{m} x}{2}\right)^{3}\right] \\
& \times\left[\frac{m \pi}{L} \sin \left(\frac{m \pi z}{L}\right) \sin \omega t+\frac{2 \pi \beta}{\lambda} \cos \left(\frac{m \pi z}{L}\right) \cos \omega t\right]
\end{aligned}
$$

which can be decomposed in two terms

$$
\Delta x^{\prime}=K_{1} x+K_{3} x^{3}
$$

where $K_{1}$ and $K_{3}$ are

$$
\begin{aligned}
K_{1} \equiv & \frac{q E_{0} \pi \Delta s}{2 m_{0} c^{2} \beta^{2} \gamma} \sum_{m} A_{m}\left[\frac{m}{L} \sin \left(\frac{m \pi z}{L}\right) \sin \omega t\right. \\
& \left.+\frac{2 \beta}{\lambda} \cos \left(\frac{m \pi z}{L}\right) \cos \omega t\right], \\
K_{3} \equiv & \frac{q E_{0} \pi \Delta s}{16 m_{0} c^{2} \beta^{2} \gamma} \sum_{m} A_{m} k_{m}^{2}\left[\frac{m}{L} \sin \left(\frac{m \pi z}{L}\right) \sin \omega t\right. \\
& \left.+\frac{2 \beta}{\lambda} \cos \left(\frac{m \pi z}{L}\right) \cos \omega t\right] .
\end{aligned}
$$

By inserting the calculated impulse into the relation (9),

$$
x_{f}^{\prime}=x_{i}^{\prime}+K_{1} x+K_{3} x^{3},
$$

which shows that a beam with linear shape in $x x^{\prime}$ phase space will change to a curve fitting a third order polynomial after passing through a cavity. We will discuss and confirm this effect by simulation in Sec. III B 6 .

The increase in the total area of projection in the $x x^{\prime}$ plane for a beam with uniform distribution will be equal to

$$
\Delta \epsilon=\int_{-x_{\max }}^{x_{\max }} \Delta x^{\prime} d x=\frac{K_{1}}{2} x^{2}+\frac{K_{3}}{4} x^{4},
$$

where $x_{\max }$ is the maximum beam radius. The last equation shows that, for a constant longitudinal phase spread and constant electric field, the emittance increases with the fourth power of the radius. In addition, since both $K_{1}$ and 
$K_{3}$ are inversely proportional to $\beta^{2} \gamma$ this effect is more dominant at low energy.

\section{Solenoids}

\section{Mathematical approach}

Equation of motion of a single particle in a solenoidal channel.-The equations of motion as function of the magnetic field are given by the Lorentz equations

$$
\frac{d \mathbf{v}}{d t}=\frac{q}{m \gamma} \mathbf{v} \times \mathbf{B},
$$

where $q$ is the charge of the particle, $m$ its mass, and $\gamma$ the relativistic factor $\gamma=1 / \sqrt{1-\beta^{2}}$. By multiplying $\mathbf{v}$ in both sides of this equation, we find that the total velocity is preserved, i.e. $v^{2}=$ const, and therefore $\gamma=$ const. The components of this equation are

$$
\begin{aligned}
\frac{d v_{x}}{d t} & =\frac{q}{m \gamma}\left(v_{y} B_{z}-v_{z} B_{y}\right), \\
\frac{d v_{y}}{d t} & =\frac{q}{m \gamma}\left(v_{z} B_{x}-v_{x} B_{z}\right), \\
\frac{d v_{z}}{d t} & =\frac{q}{m \gamma}\left(v_{x} B_{y}-v_{y} B_{x}\right) .
\end{aligned}
$$

As inside the solenoidal channel there is the vacuum, the magnetic field should satisfy the Maxwell equations

$$
\nabla \cdot \mathbf{B}=0, \quad \nabla \times \mathbf{B}=0 .
$$

As a solenoidal channel is longitudinally rotationally symmetric, by using Eq. (15) the components of the magnetic field $B_{r}$ and $B_{z}$ can be retrieved as (see Ref. [14])

$$
B_{z}=\sum_{n=0}^{\infty} \tilde{b}_{n}^{(z)} r^{2 n}
$$

and

$$
B_{r}=\sum_{n=1}^{\infty} \tilde{b}_{n}^{(r)} r^{2 n-1}
$$

where $r=\sqrt{x^{2}+y^{2}}$ and the coefficients $\tilde{b}_{n}^{(z)}$ and $\tilde{b}_{n}^{(r)}$ are given by

$$
\begin{aligned}
& \tilde{b}_{n}^{(z)}=\frac{1}{2^{2 n}} \frac{(-1)^{n}}{(n !)^{2}} \frac{\partial^{2 n}}{\partial z^{2 n}} B_{z}(0, z), \\
& \tilde{b}_{n}^{(r)}=\frac{1}{2^{2 n-1}} \frac{(-1)^{n}}{n !(n-1) !} \frac{\partial^{2 n-1}}{\partial z^{2 n-1}} B_{z}(0, z) .
\end{aligned}
$$

Note that in general $B_{z}(r, z)$, i.e. $\tilde{b}_{n}^{(r)}, \tilde{b}_{n}^{(z)}$, depends on $z$ as the properties of the solenoidal channel may vary along $z$. The condition of linear solenoidal channel is obtained when $\tilde{b}_{n}^{(z)}=0$ for $n>0$ and $\tilde{b}_{n}^{(r)}=0$ for $n>1$. In this case we obtain

$$
B_{r}=\tilde{b}_{1}^{(r)} r=-\frac{r}{2} \frac{\partial}{\partial z} B_{z}(0, z), \quad B_{z}=\tilde{b}_{0}^{(z)}=B_{z}(0, z),
$$

that is the transverse radial component of the magnetic field is simply linear. Clearly as a solenoid is never infinitely long at the entrance or exit of it, the higher order derivative of $B_{z}(0, z)$ will play a significant role, therefore making the solenoid nonlinear. Substituting the expression of Eqs. (16) and (17) into Eq. (14) and rearranging the indexes, we find the equations of motion for a particle in a general solenoidal channel:

$$
\begin{aligned}
\frac{d v_{x}}{d t}= & \frac{q}{m \gamma}\left[v_{y} \tilde{b}_{0}^{(z)}-v_{z} \tilde{b}_{1}^{(r)} y+\sum_{n=1}^{\infty}\left(v_{y} \tilde{b}_{n}^{(z)}-v_{z} \tilde{b}_{n+1}^{(r)} y\right) r^{2 n}\right] \\
\frac{d v_{y}}{d t}= & \frac{q}{m \gamma}\left[-v_{x} \tilde{b}_{0}^{(z)}+v_{z} \tilde{b}_{1}^{(r)} x\right. \\
& \left.+\sum_{n=1}^{\infty}\left(-v_{x} \tilde{b}_{n}^{(z)}+v_{z} \tilde{b}_{n+1}^{(r)} x\right) r^{2 n}\right] \\
\frac{d v_{z}}{d t}= & \frac{q}{m \gamma} \sum_{n=0}^{\infty} \tilde{b}_{n+1}^{(r)}\left(v_{x} y-v_{y} x\right) r^{2 n} .
\end{aligned}
$$

In this form the first two terms of the first two equations give the linear part of the motion. Now we perform the change of variable $d s=v_{z} d t$ and find

$$
\frac{d v_{x}}{d t}=v_{x}^{\prime} v_{z}=x^{\prime \prime} v_{z}^{2}+x^{\prime} \frac{d v_{z}}{d t},
$$

a similar expression holds for $\boldsymbol{v}_{y}^{\prime}$. Here we define ()$^{\prime}=$ $d() / d s$. Now by using Eq. (21) and making use of the definitions

$$
b_{n}^{(r)}=\frac{q}{m \gamma v_{z}} \tilde{b}_{n}^{(r)}, \quad b_{n}^{(z)}=\frac{q}{m \gamma v_{z}} \tilde{b}_{n}^{(z)},
$$

we can rewrite the first two equations of Eq. (20) as

$$
\begin{aligned}
x^{\prime \prime}= & b_{0}^{(z)} y^{\prime}-b_{1}^{(r)} y+\sum_{n=1}^{\infty}\left(b_{n}^{(z)} y^{\prime}-b_{n+1}^{(r)} y\right) r^{2 n} \\
& -\left[\sum_{n=0}^{\infty} b_{n+1}^{(r)} r^{2 n}\right] x^{\prime}\left(x^{\prime} y-y^{\prime} x\right),
\end{aligned}
$$

$$
\begin{aligned}
y^{\prime \prime}= & -b_{0}^{(z)} x^{\prime}+b_{1}^{(r)} x+\sum_{n=1}^{\infty}\left(-b_{n}^{(z)} x^{\prime}+b_{n+1}^{(r)} x\right) r^{2 n} \\
& -\left[\sum_{n=0}^{\infty} b_{n+1}^{(r)} r^{2 n}\right] y^{\prime}\left(x^{\prime} y-y^{\prime} x\right) .
\end{aligned}
$$

where $b_{n}^{(r)}, b_{n}^{(z)}$ depends on the magnetic field, and $v_{z}$ which can be obtained from the relation $v^{2}=v_{x}^{2}+v_{y}^{2}+$ $v_{z}^{2}$ as

$$
v_{z}=\frac{|v|}{\sqrt{1+x^{\prime 2}+y^{\prime 2}}} .
$$

In this form the equations of motion are more transparent 
for a discussion on the consequences of the different terms of the solenoidal field. When the solenoidal channel is nonlinear, that is $b_{n+1}^{(r)}, b_{n}^{(z)} \neq 0$ for $n=1,2,3, \ldots$, then the particle motion is nonlinear and therefore generally the transport of a beam will increase the rms emittances. When the solenoidal channel is linear, i.e. $b_{n+1}^{(r)}, b_{n}^{(z)}=0$ for $n=$ $1,2,3, \ldots$ or the beam is so small that these nonlinear components do not play a significant role, then the equations of motion become

$$
\begin{aligned}
& x^{\prime \prime}=b_{0}^{(z)} y^{\prime}-b_{1}^{(r)} y-b_{1}^{(r)} x^{\prime}\left(x^{\prime} y-y^{\prime} x\right), \\
& y^{\prime \prime}=-b_{0}^{(z)} x^{\prime}+b_{1}^{(r)} x-b_{1}^{(r)} y^{\prime}\left(x^{\prime} y-y^{\prime} x\right) .
\end{aligned}
$$

Note that now it is not possible to describe the motion via coupled linear equations as a function of only one parameter depending only on the solenoidal channel properties. The nonlinear character of Eq. (25) makes it difficult to use a Larmor transformation and decouple the system. It is also not possible to perform a generalized Edward-Teng transformation [15] as the terms $x^{\prime} y-y^{\prime} x$ in Eq. (25) are nonlinear and the dependence of $b_{0}^{(z)}$ and $b_{1}^{(r)}$ from $v_{z}$ adds a nonlinear coupling between transverse and longitudinal planes, i.e., via Eq. (24), couple even more nonlinearly the transverse planes. The nonlinear feature of Eq. (25) is the main responsible of emittance increase when a beam is transported in a solenoidal channel. However, if $x^{\prime}, y^{\prime} \rightarrow 0$ (para-axial approximation) the last term on the left-hand side of Eq. (25) is negligible and Eq. (24) shows that $v_{z} \rightarrow$ $v$ : therefore when $x^{\prime}, y^{\prime} \sim 0$ we recover the standard equation of motion studied in [16] where $b_{1}^{(r)}=-\frac{1}{2} b_{0}^{(z) \prime}$. Whether the para-axial condition is met depends on the type of solenoidal channel and how large the beam is. For example, if $x^{\prime}$ is small, but $y$ is large, then the Larmor transformation is again not possible and we expect an emittance increase. If the beam is very large, then the para-axial condition is reached with a more strict condition on how small $b_{1}^{(r)}$ should be. This means that the transport of a large beam with a large divergence in a linear solenoidal channel will always happen (at the entrance and exit) in a regime where the Larmor transformation is not possible and emittance increase is expected as a result of a nonlinear $x-y$ coupling.

Single particle transport in a linear solenoidal channel in the para-axial condition.- - In [16] the transport of a particle in an arbitrary linear solenoidal channel in a para-axial regime is solved, that is for a single particle obeying the equations of motion

$$
x^{\prime \prime}=b_{0}^{(z)} y^{\prime}+\frac{b_{0}^{(z) \prime}}{2} y \quad y^{\prime \prime}=-b_{0}^{(z)} x^{\prime}-\frac{b_{0}^{(z) \prime}}{2} x .
$$

A single particle transport in a general solenoidal channel of a particle with initial coordinate $X_{0}=\left(x_{0}, x_{0}^{\prime}, y_{0}, y_{0}^{\prime}\right)$ at $s=0$ to $X_{1}=\left(x, x^{\prime}, y, y^{\prime}\right)$ at the longitudinal position $s_{1}$ is given by

$$
X_{1}=\Sigma X_{0}
$$

where the matrix $\Sigma=\Sigma(L)$ is defined as

$$
\Sigma(\mathrm{L})=\left(\begin{array}{cc}
\operatorname{ReL} & -\mathrm{ImL} \\
\operatorname{ImL} & \operatorname{ReL}
\end{array}\right)
$$

where ReL $=\mathfrak{c}_{1} \mathrm{M}+\mathfrak{s}_{1}\left(\mathrm{M}_{i 1}+\mathrm{M}_{i 2}\right)+\mathfrak{c}_{1} \mathrm{M}_{r}$, and $-\mathrm{ImL}=$ $\mathfrak{s}_{1} \mathrm{M}-\mathfrak{c}_{1}\left(\mathrm{M}_{i 1}+\mathrm{M}_{i 2}\right)+\mathfrak{s}_{1} \mathrm{M}_{r}$, with $\mathfrak{s}_{1}=\sin \phi_{1}, \quad \mathfrak{c}_{1}=$ $\cos \phi_{1}$, and

$$
\mathrm{M}=\left(\begin{array}{cc}
\sqrt{\frac{\beta_{1}}{\beta_{0}}}\left(\mathrm{c}_{1}+\alpha_{0} \mathrm{~s}_{1}\right) & \sqrt{\beta_{1} \beta_{0}} \mathrm{~s}_{1} \\
\frac{\left(\alpha_{0}-\alpha_{1}\right) \mathrm{c}_{1}-\left(1+\alpha_{1} \alpha_{0}\right) \mathrm{s}_{1}}{\sqrt{\beta_{1} \beta_{0}}} & \sqrt{\frac{\beta_{0}}{\beta_{1}}}\left(\mathrm{c}_{1}-\alpha_{1} \mathrm{~s}_{1}\right)
\end{array}\right),
$$

$\mathrm{M}_{i 1}=\left(\begin{array}{cc}0 & 0 \\ \sqrt{\frac{\beta_{0}}{\beta_{1}}} \phi_{0}^{\prime}\left(\mathrm{c}_{1}-\alpha_{1} \mathrm{~s}_{1}\right)-\sqrt{\frac{\beta_{1}}{\beta_{0}}} \phi_{1}^{\prime}\left(\mathrm{c}_{1}+\alpha_{0} \mathrm{~s}_{1}\right) & 0\end{array}\right)$,

$\mathrm{M}_{i 2}=\left(\begin{array}{cc}\sqrt{\beta_{1} \beta_{0}} \phi_{0}^{\prime} \mathrm{s}_{1} & 0 \\ 0 & -\sqrt{\beta_{1} \beta_{0}} \phi_{1}^{\prime} \mathrm{s}_{1}\end{array}\right)$,

$\mathrm{M}_{r}=\left(\begin{array}{cc}0 & 0 \\ \phi_{0}^{\prime} \phi_{1}^{\prime} \sqrt{\beta_{1} \beta_{0}} \mathrm{~s}_{1} & 0\end{array}\right)$.

In Eqs. (29) and (30) the quantities $\mathbf{C}_{1}, \mathbf{s}_{1}$ are defined as $\mathrm{C}_{1}=\cos \psi_{1}, \mathrm{~S}_{1}=\sin \psi_{1}$. The Larmor angle $\phi$ is given by

$$
\phi(s)=\frac{1}{2} \int_{0}^{s} b_{0}^{(z)}(t) d t
$$

where $b_{0}^{(z)}(z)$ is the strength of the linear component of the solenoidal channel. The Twiss parameters $\beta, \alpha$, created by the solenoid, can be computed via the following equations:

$$
\begin{gathered}
\frac{1}{2} \beta \beta^{\prime \prime}-\frac{1}{4} \beta^{\prime 2}+\left[\frac{b_{0}^{(z)}}{2} \beta\right]^{2}=1, \quad \alpha=-\frac{\beta^{\prime}}{2}, \\
\psi=\int_{0}^{s} \frac{1}{\beta(t)} d t,
\end{gathered}
$$

with $\alpha_{0}, \beta_{0}$ the arbitrary initial Twiss parameters and $\alpha_{1}$, $\beta_{1}$ the final values.

Beam transport in a general linear solenoidal channel in the para-axial regime.-We now want to characterize how the particle distribution is transformed when transported through an arbitrary linear solenoidal channel in a paraaxial regime. At the beginning of the channel, at $s_{0}=0$, there is no solenoidal field as the beam is still out of the first solenoid: therefore $b_{0}^{(z)}\left(s_{0}\right)=0$. At the end of the solenoidal channel, in the longitudinal position $s_{1}$, the beam has gone through the last solenoid and consequently $b_{0}^{(z)}\left(s_{1}\right)=$ 0 . As $\phi^{\prime}(s)=b_{0}^{(z)}(s) / 2$, we find $\phi_{0}^{\prime}=b_{0}^{(z)}\left(s_{0}\right)=0$ and $\phi_{1}^{\prime}=b_{0}^{(z)}\left(s_{1}\right)=0$ and therefore the matrix $\Sigma(\mathrm{L})$ simplifies to

$$
\tilde{\Sigma}=\left(\begin{array}{cc}
\mathfrak{c}_{1} M & \mathfrak{S}_{1} M \\
-\mathfrak{S}_{1} M & \mathfrak{c}_{1} M
\end{array}\right)
$$

where we have omitted the dependence from $L$ and added a 
tilde to note that this result is for the special case of the general solenoidal channel (front end of the solenoidal channel). Note that $\operatorname{det} \mathrm{M}=1$, and therefore for Eq. (33) we find $\operatorname{det} \tilde{\Sigma}=1$. The beam particle distribution transforms according to

$$
\rho_{1}(X)=\rho_{0}\left(\tilde{\Sigma}^{-1} X\right)
$$

and the matrix of the second order moments transforms as

$$
\left\langle X X^{T}\right\rangle_{1}=\tilde{\Sigma}\left\langle X X^{T}\right\rangle_{0} \tilde{\Sigma}^{T},
$$

where the indices $0 / 1$ refer to the beginning/end of the solenoidal channel. The symbol ()$^{T}$ means transpose of a matrix or a vector. Now we define the submatrices

$$
\begin{gathered}
\tilde{\Sigma}_{x}=\left(\begin{array}{cc}
\left\langle x^{2}\right\rangle & \left\langle x x^{\prime}\right\rangle \\
\left\langle x x^{\prime}\right\rangle & \left\langle x^{\prime 2}\right\rangle
\end{array}\right), \quad \tilde{\Sigma}_{y}=\left(\begin{array}{cc}
\left\langle y^{2}\right\rangle & \left\langle y y^{\prime}\right\rangle \\
\left\langle y y^{\prime}\right\rangle & \left\langle y^{\prime 2}\right\rangle
\end{array}\right), \\
\tilde{\Sigma}_{x y}=\left(\begin{array}{cc}
\langle x y\rangle & \left\langle x y^{\prime}\right\rangle \\
\left\langle x^{\prime} y\right\rangle & \left\langle x^{\prime} y^{\prime}\right\rangle
\end{array}\right) .
\end{gathered}
$$

With this notation the second order moments matrix can be written as a blocks matrix as follows:

$$
\left\langle X X^{T}\right\rangle=\left(\begin{array}{cc}
\tilde{\Sigma}_{x} & \tilde{\Sigma}_{x y} \\
\tilde{\Sigma}_{x y}^{T} & \tilde{\Sigma}_{y}
\end{array}\right)
$$

and Eq. (35) can be written as

$$
\begin{aligned}
\left(\begin{array}{cc}
\tilde{\Sigma}_{x, 1} & \tilde{\Sigma}_{x y, 1} \\
\tilde{\Sigma}_{x y, 1}^{T} & \tilde{\Sigma}_{y, 1}
\end{array}\right)= & \left(\begin{array}{cc}
\mathfrak{c}_{1} \mathrm{M} & \mathfrak{S}_{1} \mathrm{M} \\
-\mathfrak{s}_{1} \mathrm{M} & \mathfrak{c}_{1} \mathrm{M}
\end{array}\right)\left(\begin{array}{cc}
\tilde{\Sigma}_{x, 0} & \tilde{\Sigma}_{x y, 0} \\
\tilde{\Sigma}_{x y, 0}^{T} & \tilde{\Sigma}_{y, 0}
\end{array}\right) \\
& \times\left(\begin{array}{cc}
\mathfrak{c}_{1} \mathrm{M}^{T} & -\mathfrak{S}_{1} \mathrm{M}^{T} \\
\mathfrak{S}_{1} \mathrm{M}^{T} & \mathfrak{c}_{1} \mathrm{M}^{T}
\end{array}\right) .
\end{aligned}
$$

Expanding this equation we find three matrical transport equations for the submatrices

$$
\begin{aligned}
\tilde{\Sigma}_{x, 1} & =\operatorname{M}\left[\mathfrak{c}_{1}^{2} \tilde{\Sigma}_{x, 0}+\mathfrak{S}_{1}^{2} \tilde{\Sigma}_{y, 0}+\mathfrak{S}_{1} \mathfrak{c}_{1}\left(\tilde{\Sigma}_{x y, 0}^{T}+\tilde{\Sigma}_{x y, 0}\right)\right] \mathrm{M}^{T} \\
\tilde{\Sigma}_{y, 1} & =\operatorname{M}\left[\mathfrak{c}_{1}^{2} \tilde{\Sigma}_{y, 0}+\mathfrak{S}_{1}^{2} \tilde{\Sigma}_{x, 0}-\mathfrak{S}_{1} \mathfrak{c}_{1}\left(\tilde{\Sigma}_{x y, 0}^{T}+\tilde{\Sigma}_{x y, 0}\right)\right] \mathrm{M}^{T} \\
\tilde{\Sigma}_{x y, 1} & =\mathrm{M}\left[-\mathfrak{S}_{1} \mathfrak{c}_{1}\left(\tilde{\Sigma}_{x, 0}-\tilde{\Sigma}_{y, 0}\right)+\mathfrak{c}_{1}^{2} \tilde{\Sigma}_{x y, 0}-\mathfrak{S}_{1}^{2} \tilde{\Sigma}_{x y, 0}^{T}\right] \mathrm{M}^{T} .
\end{aligned}
$$

These equations allow one to find the relation between the submatrices at the end of the solenoidal channel from the submatrices given at the beginning.

Transport of an uncoupled axisymmetric beam.-In this case we consider the transport of a beam at the entrance of the solenoidal channel with the following characteristics:

$$
\tilde{\Sigma}_{x, 0}=\tilde{\Sigma}_{y, 0}, \quad \tilde{\Sigma}_{x y, 0}=0 .
$$

By using these conditions in Eq. (39) we find

$$
\tilde{\Sigma}_{x, 1}=\mathrm{M} \tilde{\Sigma}_{x, 0} \mathbf{M}^{T} \quad \tilde{\Sigma}_{y, 1}=\mathrm{M} \tilde{\Sigma}_{y, 0} \mathbf{M}^{T} \quad \tilde{\Sigma}_{x y, 1}=0 .
$$

Note that in these equations the Larmor's angle $\phi$ disappears. At this point we can compute the rms emittances as determinant of a matrix. We find

$$
\begin{aligned}
& \left(\epsilon_{x, 1}\right)^{2}=\operatorname{det}\left[\tilde{\Sigma}_{x, 1}\right]=\operatorname{det}\left[\mathrm{M} \tilde{\Sigma}_{x, 0} \mathrm{M}^{T}\right]=\operatorname{det}\left[\tilde{\Sigma}_{x, 0}\right]=\left(\boldsymbol{\epsilon}_{x, 0}\right)^{2} \\
& \left(\epsilon_{y, 1}\right)^{2}=\operatorname{det}\left[\tilde{\Sigma}_{y, 1}\right]=\operatorname{det}\left[\mathrm{M} \tilde{\Sigma}_{y, 0} \mathrm{M}^{T}\right]=\operatorname{det}\left[\tilde{\Sigma}_{y, 0}\right]=\left(\epsilon_{y, 0}\right)^{2} .
\end{aligned}
$$

The result is very general: The front-end transport of an uncoupled beam (i.e. $\tilde{\Sigma}_{x, 0}=\tilde{\Sigma}_{y, 0}$ and $\tilde{\Sigma}_{x y, 0}=0$ ) in the para-axial regime in an arbitrary linear solenoidal channel leaves the transverse rms emittances at the exit $\epsilon_{x, 1}$ and $\epsilon_{y, 1}$ unchanged. No matter how $B_{z}(0, z)$ varies inside the solenoidal channel, the relation between the initial and final rms emittances remains $\epsilon_{x, 1}=\epsilon_{x, 0}$ and $\epsilon_{y, 1}=\epsilon_{y, 0}$. This result holds only when the transport is made in a condition of para-axiality and for a linear solenoidal channel. When one of these two conditions is missing then the rms emittance of the beam after the transport will increase with respect to its initial value. Note that even for a linear solenoid an emittance growth may occur if the para-axial condition is broken. That may happen when the linear fringe is too strong, or when the beam has too large a divergence.

\section{Another analytical approach}

A simplified analytical approach consists in solving the equations of motion of a single particle through a linearized solenoid. We suppose $B_{z}(z)$ increasing linearly from zero to its nominal value over a distance equal to one-third of the solenoid bore radius, Fig. 5 , constant at value $B_{0}$ inside the solenoid, finally decreasing to zero over the same fringe field length as the entrance. The absence of magnetic monopoles $(\nabla \cdot \mathbf{B}=\mathbf{0})$ and the cylindrical symmetry of the field

$$
\frac{\partial}{\partial \phi} \mathbf{B}=\mathbf{0}
$$

allows one to calculate the radial and longitudinal magnetic field in the fringe field area as

$$
B_{z}(0, z)=\frac{3}{R} z B_{0}, \quad B_{\rho}(\rho, z)=-\frac{\rho}{2} \frac{3}{R} B_{0},
$$

where $R$ is the solenoid's radius, $\rho$ is the distance from the axis, and $z$ is the longitudinal distance from the beginning of the fringe field. Because of symmetry, these expansions are correct up to the second order of $\rho$ [17]. Using Eq. (44) it is possible to write the equations of motion

$$
\frac{d \mathbf{p}}{d t}=q \mathbf{v} \times \mathbf{B},
$$

and obtain for the azimuthal plane: 


$$
\Delta p_{\phi} \cong q \int_{0}^{t} v_{z} B_{\rho}-v_{0 \rho} B_{z} d t
$$

where $t$ is the time needed for the particle to travel inside the magnetic field. In the magnetic fields the total energy is a constant of motion, i.e. $\gamma=$ const, while momentum can be exchanged among different planes, but the effect on the longitudinal plane is negligible and to a very good approximation $p_{z}$ is constant, e.g., in LINAC4's LEBT, $p_{f z}=$ $p_{i z}\left(1-\delta p_{z}\right)$ where $\delta p_{z} \sim 10^{-7}$ and $p_{i z}$ and $p_{f z}$ are the initial and final longitudinal momentum, respectively. Now the change in azimuthal momentum, equal to the final azimuthal momentum of the particle, can be calculated as

$$
\Delta p_{\phi}=-q \int_{0}^{z=R / 3} B_{\rho} d z+q \frac{p_{0 \rho}}{p_{z}} \int_{0}^{z=R / 3} B_{z} d z .
$$

By inserting the values of fields and integrating we find

$$
p_{\phi(f 1)}=\Delta p_{\phi}=q B_{0}\left(\frac{R}{3} \frac{p_{0 \rho}}{p_{z}}+\frac{\rho_{0}}{2}\right),
$$

where $\rho_{0}$ is the initial beam radius, $p_{0 \rho}$ is initial radial momentum, and $p_{\phi(f 1)}$ is the azimuthal momentum at the end of the entrance fringe field. Since we assumed a constant longitudinal momentum, we can write the momentum conservation relation in the transverse plane,

$$
p_{\rho}^{2}+p_{\phi}^{2}=p_{0 \rho}^{2} .
$$

The momentum values at the entrance of the constant field are known, Eqs. (48) and (49) and $p_{z}$ is constant, now the remaining two coupled differential equations in transverse planes can be solved, resulting in

$$
\begin{aligned}
p_{\rho(\mathrm{sol})}= & \left(p_{0 \rho}^{2}-p_{\phi}^{2}\right)^{1 / 2} \cos \left(\frac{B_{0}}{m \gamma} \frac{s}{v_{z}}\right)-q B_{0}\left(\frac{\rho_{0}}{2}+\frac{R}{3} \frac{p_{0 \rho}}{p_{z}}\right) \\
& \times \sin \left(\frac{B_{0}}{m \gamma} \frac{s}{v_{z}}\right), \\
p_{\phi(\mathrm{sol})}= & \left(p_{0 \rho}^{2}-p_{\phi}^{2}\right)^{1 / 2} \sin \left(\frac{B_{0}}{m \gamma} \frac{s}{v_{z}}\right)+q B_{0}\left(\frac{\rho_{0}}{2}+\frac{R}{3} \frac{p_{0 \rho}}{p_{z}}\right) \\
& \times \cos \left(\frac{B_{0}}{m \gamma} \frac{s}{v_{z}}\right),
\end{aligned}
$$

where $s$ is the length of the uniform field of the solenoid, and the suffix sol refers to values at the end of the solenoid's constant field. In the exit fringe field everything is similar to the entrance fringe field, except that the longitudinal field decreases from its nominal value to zero over the same length. Taking this into account and using the same procedure we reach

$$
\Delta p_{\phi(f 2)}=q B_{0}\left(\frac{R}{3} \frac{p_{\rho}}{p_{z}}-\frac{\rho}{2}\right)
$$

where $p_{\rho}$ is the radial momentum at the end of constant
TABLE I. Physical properties of devices.

\begin{tabular}{lcc}
\hline \hline & Field map length $(\mathrm{mm})$ & Aperture radius $(\mathrm{mm})$ \\
\hline rf cavity & 160 & 20 \\
Solenoid & 240 & 30 \\
\hline \hline
\end{tabular}

field inside the solenoid, $\rho$ is the radius of the particle at this point, and $\Delta p_{\phi(f 2)}$ is the azimuthal momentum change at the exit fringe field. By dividing the azimuthal kicks into two parts, one due to the divergence and another due to the radius of the particle, Eqs. (48) and (51), show that the component of the azimuthal kick imparted due to the distance of particle from the design orbit has opposite signs in the entrance and exit fringe fields which could be to some extent canceled out, while the components which are due to the divergence, $p_{\rho} / p_{z}$, have the same sign and add. All these observations show that the lower the divergence when entering the solenoid, the lower the emittance growth when exiting the solenoid.
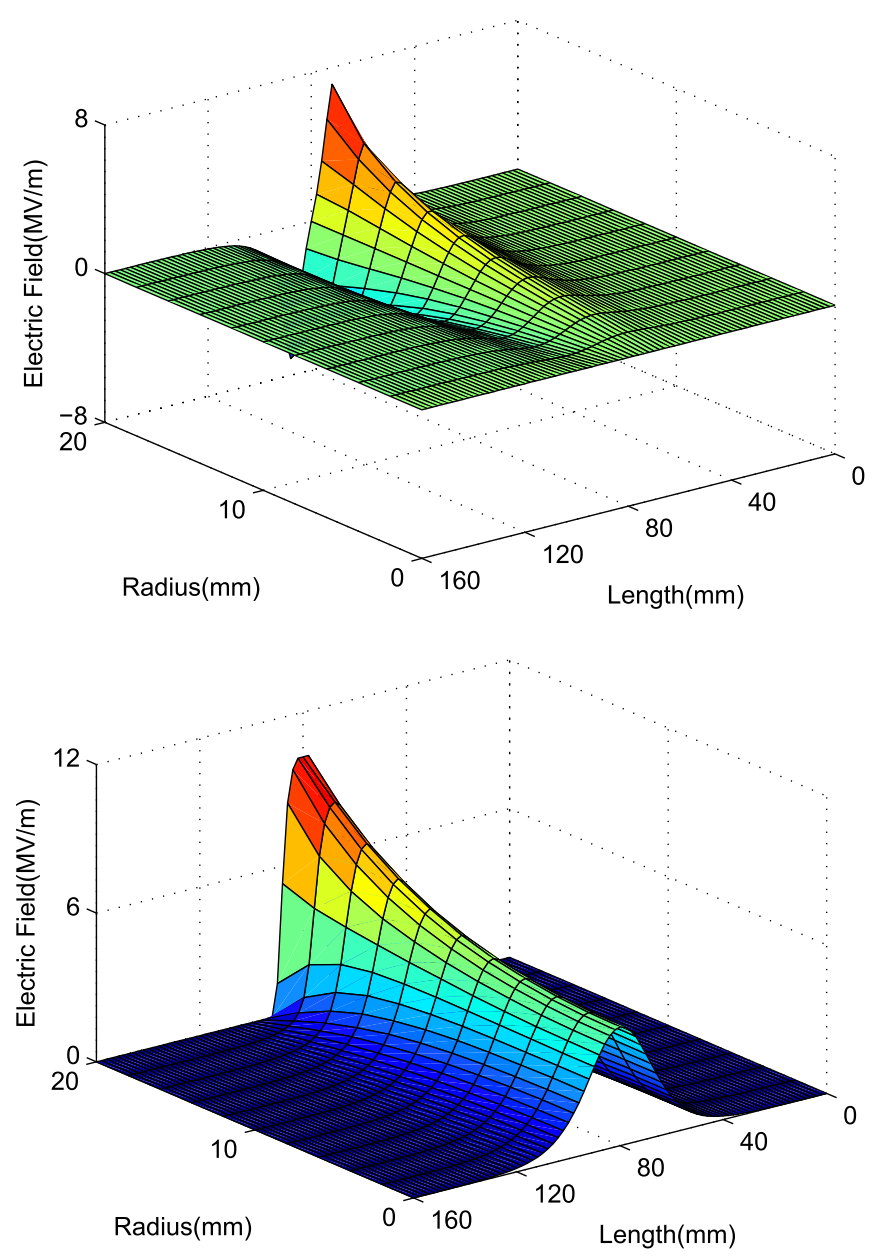

FIG. 4. (Color) 3D electric fields used in simulations. Top: Radial electric field. Bottom: longitudinal electric field. 

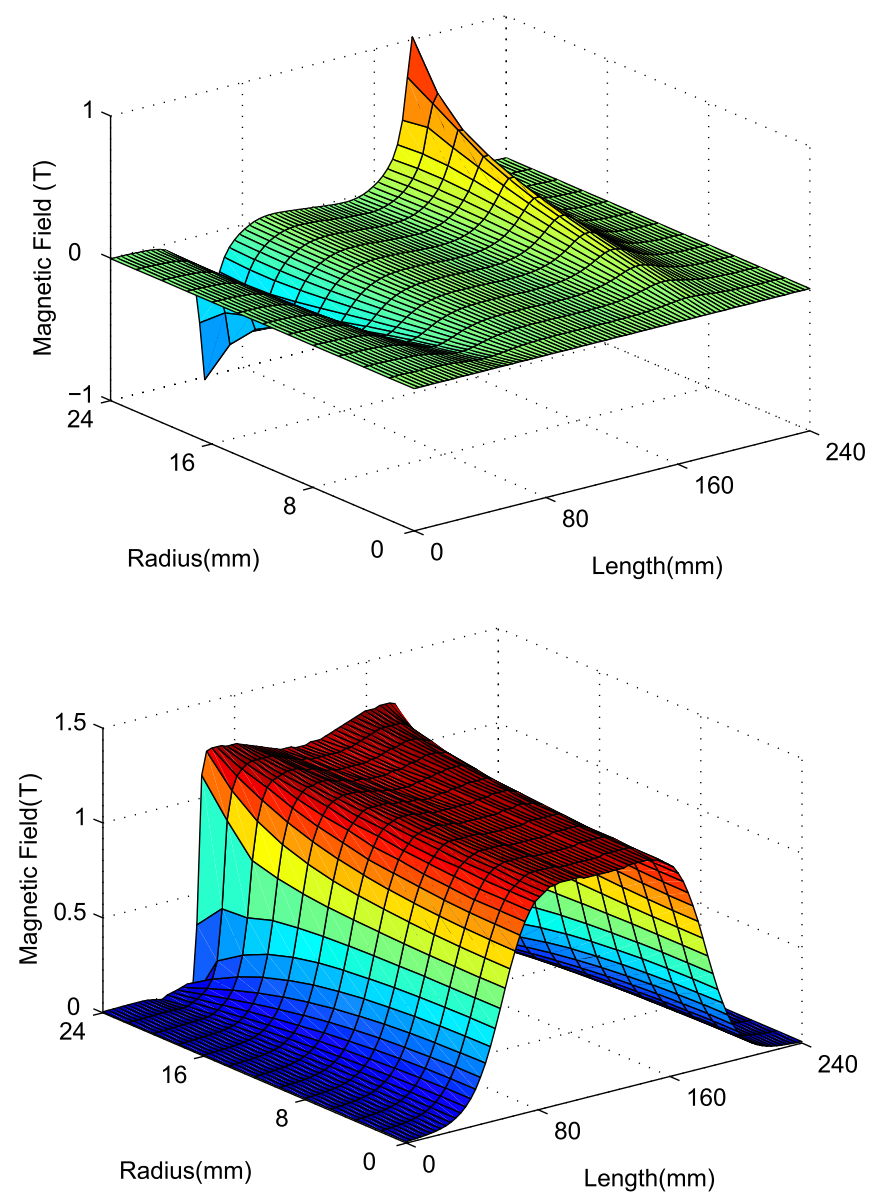

FIG. 5. (Color) 3D magnetic fields used in simulations. Top: radial magnetic field. Bottom: longitudinal magnetic field.

\section{SIMULATION}

\section{A. Simulation tools}

In order to confirm the analytic results we integrated the equations of motion of a particle beam passing through a rf cavity or a solenoid field map under varying initial conditions and we followed the evolution of the emittance and halo parameters. The beam dynamics was calculated with the programs PATHMANAGER [18] and TRACEWIN [19]. Both programs have the ability to import an electric or magnetic field map. For the examples reported in the following we have used a buncher cavity as designed for the $3 \mathrm{MeV}$ LINAC4 chopper line [20] and a solenoid as used for the matching from the source to the RFQ in LINAC4. The rf cavity (buncher) is a $352 \mathrm{MHz} \mathrm{TM}_{010}$ resonator, with a length of $160 \mathrm{~mm}$ and a bore radius of $20 \mathrm{~mm}$. The maximum voltage is $150 \mathrm{kV}$ [20]. The solenoid is $190 \mathrm{~mm}$ long and has a bore radius of $30 \mathrm{~mm}$ [21]. Field maps were created by SUPERFISH [22] which is a 2D Poisson equation solver developed at Los Alamos National Laboratory. Field maps are shown in Figs. 4 and 5 for the nominal values.

To avoid all the effects other than the rf cavity or solenoid in each simulation, we have chosen a setup, Table I, which includes just the field map.

\section{B. Simulation results}

\section{1. rf cavities}

In order to correlate the evolution of the emittance when traversing a rf cavity with beam parameters at the entrance (transverse size, transverse aspect ratio, and divergence), we have generated beams composed of $10^{5}$ macroparticles with a total current of $70 \mathrm{~mA}$, and an average energy of $3 \mathrm{MeV}(\beta \gamma \simeq 0.08)$, which at $352 \mathrm{MHz}$, the frequency of machine, gives a $\beta \lambda=0.068 \mathrm{~m}$. The transverse input beam parameters have been varied whereas phase and energy spread have been kept constant in all cases. Each case was run with a uniform and a Gaussian distribution truncated at $3 \times$ standard deviation, $\sigma$. The contribution of space charge has been separately evaluated. At the entrance of the rf cavity we had a set of simulated beams which is shown in Table II.

\section{Space-charge effect evaluation [v(voltage $)=0$ ]}

Space charge is one of the intensity limitations in ion LINACs, especially at low energies where it is not com-

TABLE II. Beam parameters in the rf cavity study.

\begin{tabular}{|c|c|c|c|c|c|c|c|}
\hline $\begin{array}{l}x_{\mathrm{rms}} \\
y_{\mathrm{rms}} \\
\end{array}$ & $\begin{array}{l}\mathrm{mm} \\
\mathrm{mm}\end{array}$ & $\begin{array}{l}2.0 \\
2.0\end{array}$ & $\begin{array}{l}4.0 \\
4.0\end{array}$ & $\begin{array}{l}5.0 \\
5.0\end{array}$ & $\begin{array}{l}2.6 \\
2.0\end{array}$ & $\begin{array}{l}2.6 \\
4.0\end{array}$ & $\begin{array}{l}2.6 \\
2.6\end{array}$ \\
\hline$\alpha_{x}$ & & 0.0 & 0.0 & 0.0 & $0.0,1.5$ & $0.0,1.5$ & $0.0,1.5,3.4,7,10,15$ \\
\hline$\beta_{x}$ & $\mathrm{~mm} / \pi \mathrm{mrad}$ & 1.07 & 4.27 & 6.67 & 1.90 & 1.90 & 1.90 \\
\hline$\beta \gamma \epsilon_{x-\mathrm{rms}}$ & $\pi \mathrm{mm} \operatorname{mrad}$ & 0.3 & 0.3 & 0.3 & 0.3 & 0.3 & 0.3 \\
\hline$\alpha_{y}$ & & 0.0 & 0.0 & 0.0 & $-\alpha_{x}$ & $-\alpha_{x}$ & $-\alpha_{x}$ \\
\hline$\beta_{y}$ & $\mathrm{~mm} / \pi \mathrm{mrad}$ & 1.07 & 4.27 & 6.67 & 1.07 & 4.27 & 1.90 \\
\hline$\beta \gamma \epsilon_{y-\mathrm{rms}}$ & $\pi \mathrm{mm} \mathrm{mrad}$ & 0.3 & 0.3 & 0.3 & 0.3 & 0.3 & 0.3 \\
\hline$\alpha_{z}$ & & 0.5 & 0.5 & 0.5 & 0.5 & 0.5 & 0.5 \\
\hline$\phi_{\mathrm{rms}} @ 352 \mathrm{MHz}$ & $\operatorname{deg}$ & 19 & 19 & 19 & 19 & 19 & 19 \\
\hline$\beta \gamma \epsilon_{z-\mathrm{rms}}$ & $\pi \mathrm{mm} \mathrm{mrad}$ & 0.4 & 0.4 & 0.4 & 0.4 & 0.4 & 0.4 \\
\hline Number of particles & $10^{5}$ & 1 & 1 & 1 & 1 & 1 & 1 \\
\hline
\end{tabular}


TABLE III. Space-charge dependence-uniform beam, $v=0$.

\begin{tabular}{lcccc}
\hline \hline$R_{\text {rms }} \mathrm{mm}$ & $\Delta \epsilon_{T}[\%]$ & $\Delta H_{T} 10^{-2}$ & $\Delta \epsilon_{Z}[\%]$ & $\Delta H_{Z} 10^{-2}$ \\
\hline 2.0 & 0.38 & 1.11 & 0.32 & 0.89 \\
2.6 & 0.36 & 0.79 & 0.23 & 0.55 \\
4.0 & 0.24 & 0.52 & 0.11 & 0.25 \\
5.0 & 0.18 & 0.04 & 0.07 & 0.14 \\
\hline \hline
\end{tabular}

TABLE IV. Space-charge dependence-Gaussian beam, $v=$ 0 .

\begin{tabular}{lcccc}
\hline \hline$R_{\text {rms }} \mathrm{mm}$ & $\Delta \epsilon_{T}[\%]$ & $\Delta H_{T} 10^{-2}$ & $\Delta \epsilon_{Z}[\%]$ & $\Delta H_{Z} 10^{-2}$ \\
\hline 2.0 & 1.0 & 2.6 & 1.3 & 4.4 \\
2.6 & 0.9 & 2.3 & 0.7 & 2.4 \\
4.0 & 0.6 & 2.0 & 0.3 & 0.8 \\
5.0 & 0.5 & 1.6 & 0.1 & 0.3 \\
\hline \hline
\end{tabular}

pensated by magnetic self-fields due to the velocity of particles. In high intensity machines, like LINAC4, its effect should be fully considered. In order to estimate the effect of space charge in our test cases we have generated a round, parallel beam with varying beam size and distribution and made it drift over a length equal to the rf cavity length. The results of these tests are reported in Table III and IV for a uniform and Gaussian beam distribution, respectively. In case of round beams subscripts $T$ and $Z$ indicate transverse and longitudinal values, respectively. The effect of space charge on transverse emittance increase in the worst case, i.e., smallest transverse size with Gaussian distribution, is less than $1 \%$, and decreases to half a percent for the biggest size beam. From this set of simulations we can conclude that the contribution of the space charge in our results is less than $1 \%$ and therefore is not relevant for the conclusions throughout this paper.

TABLE V. Beam size dependence-uniform beam, $v=$ $340 \mathrm{kV}$.

\begin{tabular}{lcccc}
\hline \hline$R_{\text {rms }} \mathrm{mm}$ & $\Delta \epsilon_{T}[\%]$ & $\Delta H_{T} 10^{-2}$ & $\Delta \epsilon_{Z}[\%]$ & $\Delta H_{Z} 10^{-2}$ \\
\hline 2.0 & 0.9 & 1.9 & 1.0 & 2.3 \\
2.6 & 1.5 & 3.0 & 1.6 & 3.6 \\
4.0 & 4.6 & 9.9 & 4.7 & 10.5 \\
5.0 & 12.5 & 29.5 & 9.6 & 22.7 \\
\hline \hline
\end{tabular}

TABLE VI. Beam size dependence-Gaussian beam, $v=$ $340 \mathrm{kV}$.

\begin{tabular}{lcccc}
\hline \hline$R_{\mathrm{rms}} \mathrm{mm}$ & $\Delta \epsilon_{T}[\%]$ & $\Delta H_{T} 10^{-2}$ & $\Delta \epsilon_{Z}[\%]$ & $\Delta H_{Z} 10^{-2}$ \\
\hline 2.0 & 1.6 & 2.9 & 1.5 & 1.8 \\
2.6 & 2.1 & 2.7 & 2.4 & 4.6 \\
4.0 & 6.4 & 15.1 & 6.8 & 14.4 \\
5.0 & 20.0 & 70.2 & 13.8 & 31.6 \\
\hline \hline
\end{tabular}

TABLE VII. Aspect ratio dependence-uniform beam $\left(\alpha_{x}=\right.$ $\left.1.5 ; \alpha_{y}=-1.5\right)$.

\begin{tabular}{lcccccr}
\hline \hline $\begin{array}{l}\text { Field } \\
\mathrm{kV}\end{array}$ & $\begin{array}{c}x_{\mathrm{rms}} \\
\mathrm{mm}\end{array}$ & $\begin{array}{l}y_{\mathrm{rms}} \\
\mathrm{mm}\end{array}$ & $\begin{array}{l}\Delta \boldsymbol{\epsilon}_{x} \\
{[\%]}\end{array}$ & $\begin{array}{c}\Delta H_{x} \\
10^{-2}\end{array}$ & $\begin{array}{l}\Delta \boldsymbol{\epsilon}_{y} \\
{[\%]}\end{array}$ & $\begin{array}{r}\Delta H_{y} \\
10^{-2}\end{array}$ \\
\hline \multirow{3}{*}{0} & & 2.0 & 0.4 & 0.8 & 0.3 & 0.7 \\
& \multirow{2}{*}{2.6} & 2.6 & 0.3 & 0.7 & 0.4 & 0.8 \\
& & 4.0 & 0.2 & 0.5 & 0.3 & 0.7 \\
\hline \multirow{3}{*}{340} & \multirow{2}{*}{2.6} & 2.0 & 1.4 & 2.8 & 1.0 & 2.0 \\
& & 2.6 & 1.1 & 2.5 & 1.8 & 3.5 \\
\hline \hline
\end{tabular}

TABLE VIII. Aspect ratio dependence-Gaussian beam $\left(\alpha_{x}=1.5 ; \alpha_{y}=-1.5\right)$.

\begin{tabular}{lcccccr}
\hline \hline $\begin{array}{l}\text { Field } \\
\mathrm{kV}\end{array}$ & $x_{\mathrm{rms}}$ & $y_{\mathrm{rms}}$ & $\Delta \epsilon_{x}$ & $\Delta H_{x}$ & $\begin{array}{l}\Delta \epsilon_{y} \\
{[\%]}\end{array}$ & $\begin{array}{r}\Delta H_{y} \\
10^{-2}\end{array}$ \\
\hline \multirow{3}{*}{0} & $\mathrm{~mm}$ & $\mathrm{~mm}$ & {$[\%]$} & $10^{-2}$ & {$[\%]$} \\
& 2.6 & 2.6 & 0.9 & 2.8 & 0.8 & 2.3 \\
& & 4.0 & 0.8 & 2.2 & 1.0 & 2.5 \\
340 & 2.6 & 2.6 & 1.3 & 1.1 & 3.6 \\
\hline & & 2.0 & 3.0 & 1.6 & 2.8 \\
& & & 1.7 & 2.7 & 2.6 & 3.5 \\
\hline \hline
\end{tabular}

TABLE IX. Aspect ratio dependence-uniform beam (parallel).

\begin{tabular}{lcccccc}
\hline \hline $\begin{array}{l}\text { Field } \\
\mathrm{kV}\end{array}$ & $x_{\mathrm{rms}}$ & $y_{\mathrm{rms}}$ & $\begin{array}{c}\Delta \epsilon_{x} \\
\mathrm{~mm}\end{array}$ & $\begin{array}{c}\Delta H_{x} \\
10^{-2}\end{array}$ & $\begin{array}{r}\Delta \epsilon_{y} \\
{[\%]}\end{array}$ & $\begin{array}{r}\Delta H_{y} \\
10^{-2}\end{array}$ \\
\hline \multirow{3}{*}{0} & & 2.0 & 0.4 & 0.9 & 0.3 & 0.6 \\
& 2.6 & 2.6 & 0.3 & 0.8 & 0.4 & 0.8 \\
& & 4.0 & 0.2 & 0.5 & 0.3 & 0.7 \\
\hline & & 2.0 & 1.6 & 3.3 & 0.8 & 1.6 \\
340 & 2.6 & 2.6 & 1.4 & 3.0 & 1.5 & 2.9 \\
& & 4.0 & 1.1 & 2.4 & 4.7 & 10.0 \\
\hline \hline
\end{tabular}

TABLE X. Aspect ratio dependence-Gaussian beam (parallel).

\begin{tabular}{lcccccr}
\hline \hline $\begin{array}{l}\text { Field } \\
\mathrm{kV}\end{array}$ & $x_{\mathrm{rms}}$ & $\begin{array}{l}y_{\mathrm{rms}} \\
\mathrm{mm}\end{array}$ & $\begin{array}{c}\Delta \boldsymbol{\epsilon}_{x} \\
\mathrm{~mm}\end{array}$ & $\begin{array}{c}\Delta H_{x} \\
10^{-2}\end{array}$ & $\begin{array}{r}\Delta \epsilon_{y} \\
{[\%]}\end{array}$ & $\begin{array}{r}\Delta H_{y} \\
10^{-2}\end{array}$ \\
\hline \multirow{3}{*}{0} & & 2.0 & 1.3 & 3.6 & 0.7 & 1.9 \\
& \multirow{2}{*}{2.6} & 2.6 & 0.9 & 2.5 & 0.8 & 2.1 \\
& & 4.0 & 0.6 & 1.5 & 0.9 & 3.2 \\
\hline & & 2.0 & 2.6 & 4.1 & 1.3 & 2.1 \\
340 & 2.6 & 2.6 & 2.1 & 2.9 & 2.0 & 2.6 \\
& & 4.0 & 1.7 & 2.1 & 6.5 & 14.6 \\
\hline \hline
\end{tabular}


TABLE XI. Divergence dependence-uniform beam $\left(R_{\mathrm{rms}}=\right.$ $2.6 \mathrm{~mm}), v=340 \mathrm{kV}$.

\begin{tabular}{lrcccc}
\hline \hline$\alpha_{x}$ & \multicolumn{1}{c}{$\alpha_{y}$} & $\Delta \epsilon_{x}[\%]$ & $\Delta H_{x} 10^{-2}$ & $\Delta \epsilon_{y}[\%]$ & $\Delta H_{y} 10^{-2}$ \\
\hline 0.0 & 0.0 & 1.4 & 3.0 & 1.5 & 2.9 \\
1.5 & -1.5 & 1.1 & 2.5 & 1.8 & 3.5 \\
3.4 & -3.4 & 0.9 & 1.9 & 2.4 & 4.3 \\
7.0 & -7.0 & 0.6 & 1.2 & 4.0 & 6.7 \\
10.0 & -10.0 & 0.4 & 0.8 & 6.3 & 9.4 \\
15.0 & -15.0 & 0.2 & 0.7 & 12.0 & 17.3 \\
\hline \hline
\end{tabular}

TABLE XII. Divergence dependence-Gaussian beam $\left(R_{\mathrm{rms}}=2.6 \mathrm{~mm}\right), v=340 \mathrm{kV}$.

\begin{tabular}{lrcccc}
\hline \hline$\alpha_{x}$ & \multicolumn{1}{c}{$\alpha_{y}$} & $\Delta \epsilon_{x}[\%]$ & $\Delta H_{x} 10^{-2}$ & $\Delta \epsilon_{y}[\%]$ & $\Delta H_{y} 10^{-2}$ \\
\hline 0.0 & 0.0 & 2.1 & 2.9 & 2.0 & 2.6 \\
1.5 & -1.5 & 1.7 & 2.7 & 2.6 & 3.5 \\
3.4 & -3.4 & 1.4 & 2.2 & 3.2 & 3.7 \\
7.0 & -7.0 & 0.9 & 1.7 & 5.1 & 6.3 \\
10.0 & -10.0 & 0.6 & 0.9 & 7.4 & 10.0 \\
15.0 & -15.0 & 0.3 & 0.9 & 14.4 & 24.2 \\
\hline \hline
\end{tabular}

\section{Dependence on beam size}

In $\mathrm{rf}$ cavities the radial component of the electric field grows quadratically with radius up to half of maximum radius of the rf cavity, whereas the longitudinal electric field increases linearly toward higher radii. As a result, the variation of the beam divergence is not linear with the radial position of the particle, therefore an increase of the emittance, proportional to the fourth power of the beam radius, is to be expected when a beam crosses a rf cavity [Eq. (12)]. In order to numerically test this effect, we have tracked beams of different transverse sizes through the rf cavity excited at the voltage of $340 \mathrm{kV}$. The simulation results are shown in Tables V and VI. Fitting a polynomial to the simulated data indicates a cubic dependence of emittance increase with radius, in complete agreement with the analytical prediction. As shown in the previous chapter the space-charge effects contribute to a maximum of $1 \%$ to the values reported in Tables V and VI; therefore going to smaller beam radii will not bring any further improvement to the transverse emittance and will instead degrade the longitudinal. In a space-charge dominated LINAC a trade-off between the effects of the rf fields (increasing with beam size) and space-charge effects (decreasing with beam size) must be carefully balanced. The halo also increases with the fourth power of beam size irrespective of the distribution of the beam; however, the grow rate is faster for Gaussian beams. We repeated the simulation of Gaussian beam with a distribution truncated at $5 \times$ standard deviation instead of $3 \times$ standard deviation, and we found that the emittance and halo increase to bigger values but still proportional to the fourth power of the beam size.

\section{Dependence on transverse aspect ratio}

We took three ellipsoidal beams to investigate the effect of aspect ratio on emittance increase and the possible effect of horizontal and vertical planes on each other. All three beams had the same convergence in the horizontal plane, $\alpha_{x}=1.5$, and the same divergence in the vertical plane, $\alpha_{y}=-1.5$, Tables VII and VIII. Lower emittance growth in the horizontal plane for bigger vertical sizes of the beam is within space-charge statistical error. If we subtract the effect of space charge there is not any significant emittance increase in the horizontal, $x$ plane, when the rf cavity is tuned on at $340 \mathrm{kV}$. Also for parallel beams with the same aspect ratio the effect of field plus space-charge effect is indicated in Tables IX and X for both uniform and Gaussian beam distributions to demonstrate more clearly the independence of vertical and horizontal planes. Based on this study we can look at each plane irrespective of the other plane, i.e., the two transverse planes behave independently of each other.

\section{Dependence on divergence}

The beam divergence strongly affects the beam behavior, and to quantify its effect we took a round beam of $2.6 \mathrm{~mm} \mathrm{rms} \mathrm{size,} \mathrm{and} \mathrm{varied} \mathrm{the} \alpha$ from 0 to the highest negative value possible without generating losses. In order
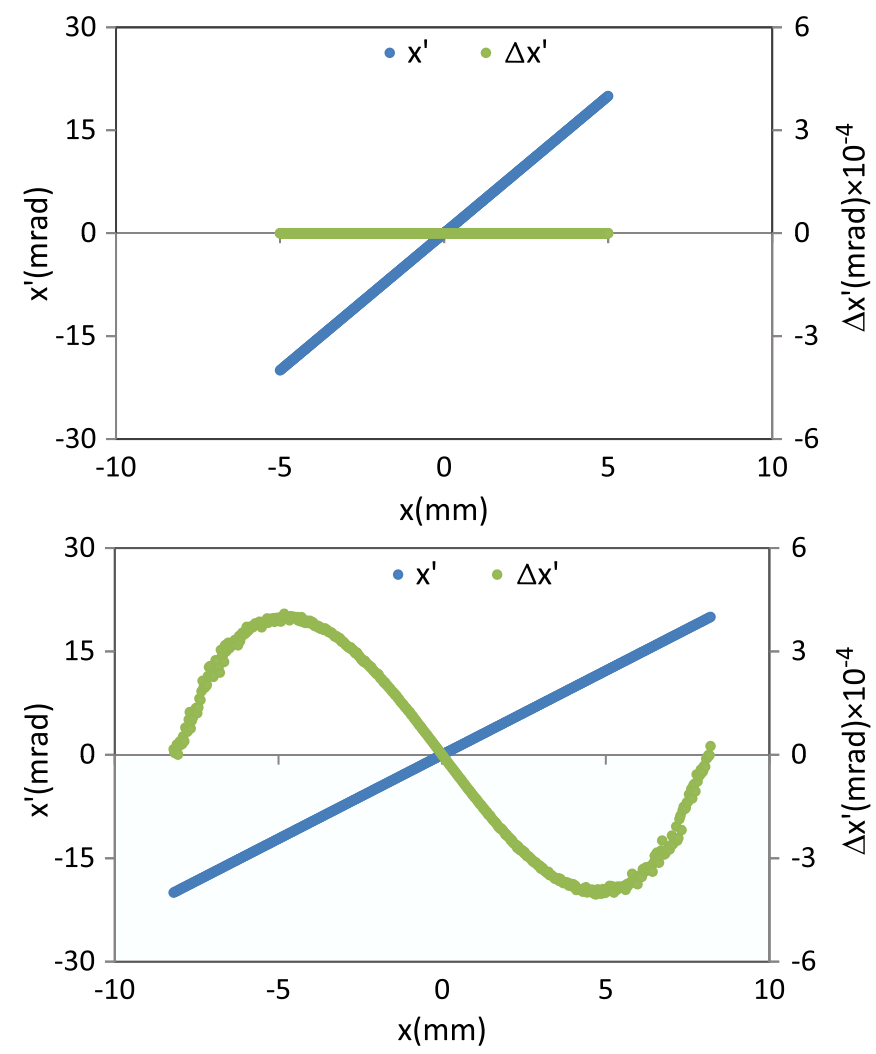

FIG. 6. (Color) Distribution in $X X^{\prime}$ phase space and difference between this distribution and fitted line. Top: initial distribution. Bottom: final distribution. 
to avoid compressing the beam volume drastically, we have compensated by increasing $\alpha$ in the other transverse plane. We found that a converging beam, $\alpha>0$, experiences less emittance growth when traversing a rf cavity than a parallel or diverging beam, Tables XI and XII. While for converging beams the beam size does not decrease linearly with the convergence, due to the presence of space-charge repulsive force, dependence of the beam size to the initial divergence is the effective factor in inducing more emittance increase for the beams with bigger divergence.

\section{Another test of emittance growth}

Another way to highlight emittance growth is to observe the deformation of a line in phase space. To this end we generated a special beam uniformly distributed in vertical plane within $\pm 4.8 \mathrm{~mm}$ and $\pm 2.7 \mathrm{mrad}$, in the longitudinal plane within $1.3 \mathrm{rad}$ and $\pm 2 \%$ of kinetic energy, and laying on a line $\left(X^{\prime}=4 \times X\right)$ in the horizontal plane.

We passed this bunch of 500 particles through a cavity with average axial field of $170 \mathrm{kV}$ without space-charge effects. After the passage through the field the particles from the line of $X^{\prime}=4 \times X$ were fitting a third order polynomial, while the evolution of a line which passes

TABLE XIII. Effect of each field component ( $v=340 \mathrm{kV})$, uniform.

\begin{tabular}{lcccc}
\hline \hline & $\Delta \epsilon_{T}[\%]$ & $\Delta H_{T} 10^{-2}$ & $\Delta \epsilon_{L}[\%]$ & $\Delta H_{L} 10^{-2}$ \\
\hline$E_{z} \neq 0, E_{r} \neq 0$ & 4.6 & 9.9 & 4.7 & 10.5 \\
$E_{r}=0$ & 0.2 & 1.1 & 4.6 & 10.3 \\
$E_{z}=0$ & 4.5 & 20.1 & 0.1 & 0.3 \\
\hline \hline
\end{tabular}

TABLE XIV. Effect of each field component ( $v=340 \mathrm{kV})$, Gaussian.

\begin{tabular}{lcccc}
\hline \hline & $\Delta \epsilon_{T}[\%]$ & $\Delta H_{T} 10^{-2}$ & $\Delta \epsilon_{L}[\%]$ & $\Delta H_{L} 10^{-2}$ \\
\hline$E_{z} \neq 0, E_{r} \neq 0$ & 6.4 & 15.1 & 6.8 & 14.4 \\
$E_{r}=0$ & 0.6 & 4.2 & 6.7 & 14.1 \\
$E_{z}=0$ & 6.3 & 30.7 & 0.3 & 0.7 \\
\hline \hline
\end{tabular}

TABLE XV. Beam parameters at solenoid study.

\begin{tabular}{lccc}
\hline \hline$\alpha_{T}$ & & 0 & -15 \\
\hline$\beta_{T}$ & $\mathrm{~mm} / \pi \mathrm{mrad}$ & 2.34 & 2.34 \\
$\epsilon_{T-\text { rms }}$ & $\pi \mathrm{mm} \mathrm{mrad}$ & 17.7 & 17.7 \\
$\alpha_{L}$ & & 0 & 0 \\
$\sigma(\Delta E / E)$ & $\%$ & 0.25 & 0.25 \\
$R_{\text {rms }}$ & $\mathrm{mm}$ & 6.4 & 6.4 \\
Number of particles & $10^{5}$ & 0.5 & 0.5 \\
Energy & $\mathrm{keV}$ & 95 & 95 \\
\hline \hline
\end{tabular}

TABLE XVI. Divergence and field dependence in solenoids.

\begin{tabular}{lrcccc}
\hline \hline Field $T$ & \multicolumn{1}{c}{$\alpha$} & $\Delta \epsilon_{T}[\%]$ & $\Delta H_{T} 10^{-1}$ & $\Delta \epsilon_{L}[\%]$ & $\Delta H_{T} 10^{-2}$ \\
\hline 0.6 & 0 & 14.8 & 2.2 & 0.9 & 2.6 \\
0.6 & -15 & 156.3 & 21.0 & 4.8 & 20.1 \\
1.08 & 0 & 92.2 & 11.3 & 9.3 & 25.3 \\
1.08 & -15 & 484.5 & 29.7 & 33.7 & 71.4 \\
\hline \hline
\end{tabular}

through a drift with the same length results to another line, $X^{\prime}=2.4384 \times X$, without any distortion.

If we plot the difference between the line obtained after the drift and the curve obtained after passing through the rf cavity, we can visualize the effect of the rf field on the particle emittance, Fig. 6.

\section{Emittance evolution in a field with selected components}

To evaluate the relative weight of the radial and longitudinal components on emittance increase, we artificially nullified each component in turn and observed the effect on a round parallel beam with transverse size equal to $R_{\mathrm{rms}}=$ $4.0 \mathrm{~mm}$. Tables XIII and XIV show that transverse emittance increase is mostly due to the radial electric field, the remaining part of transverse emittance increase in the absence of radial field is due to space-charge effect, Tables IV and V.
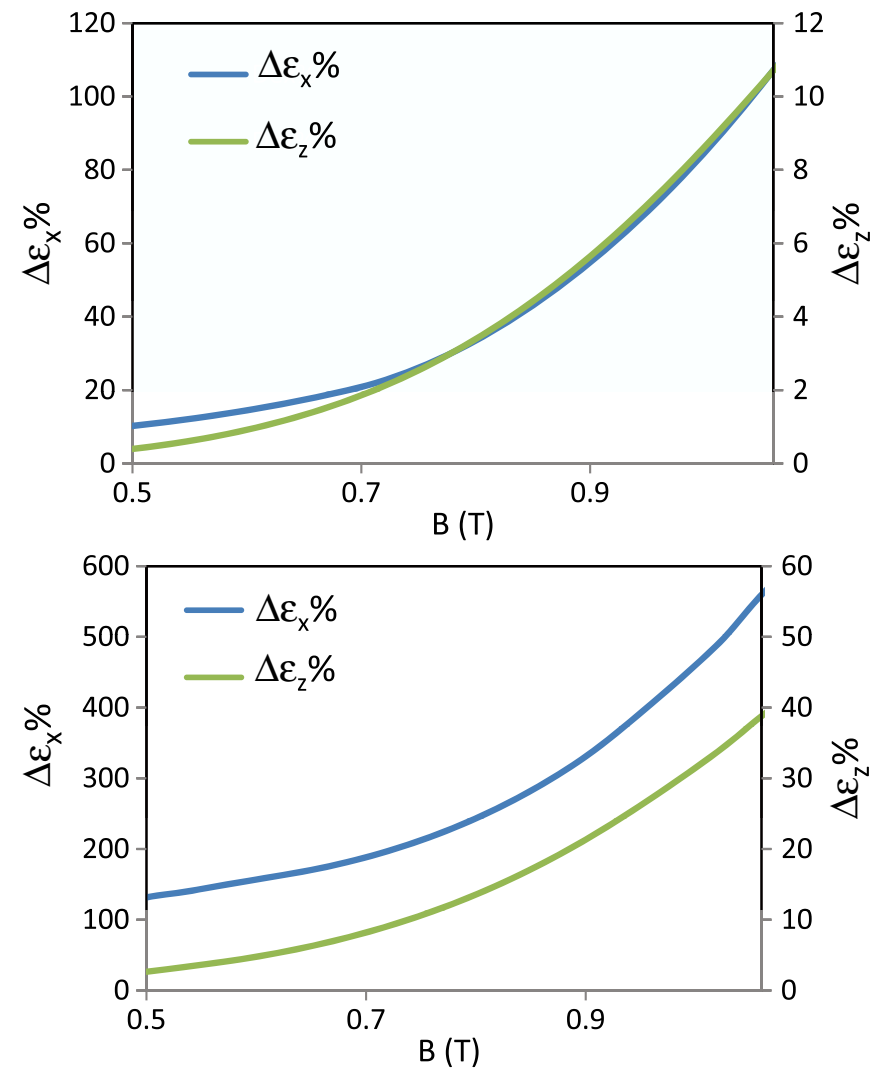

FIG. 7. (Color) Emittance evolution as a function of applied field. Top: parallel initial beam. Bottom: diverging initial beam. 

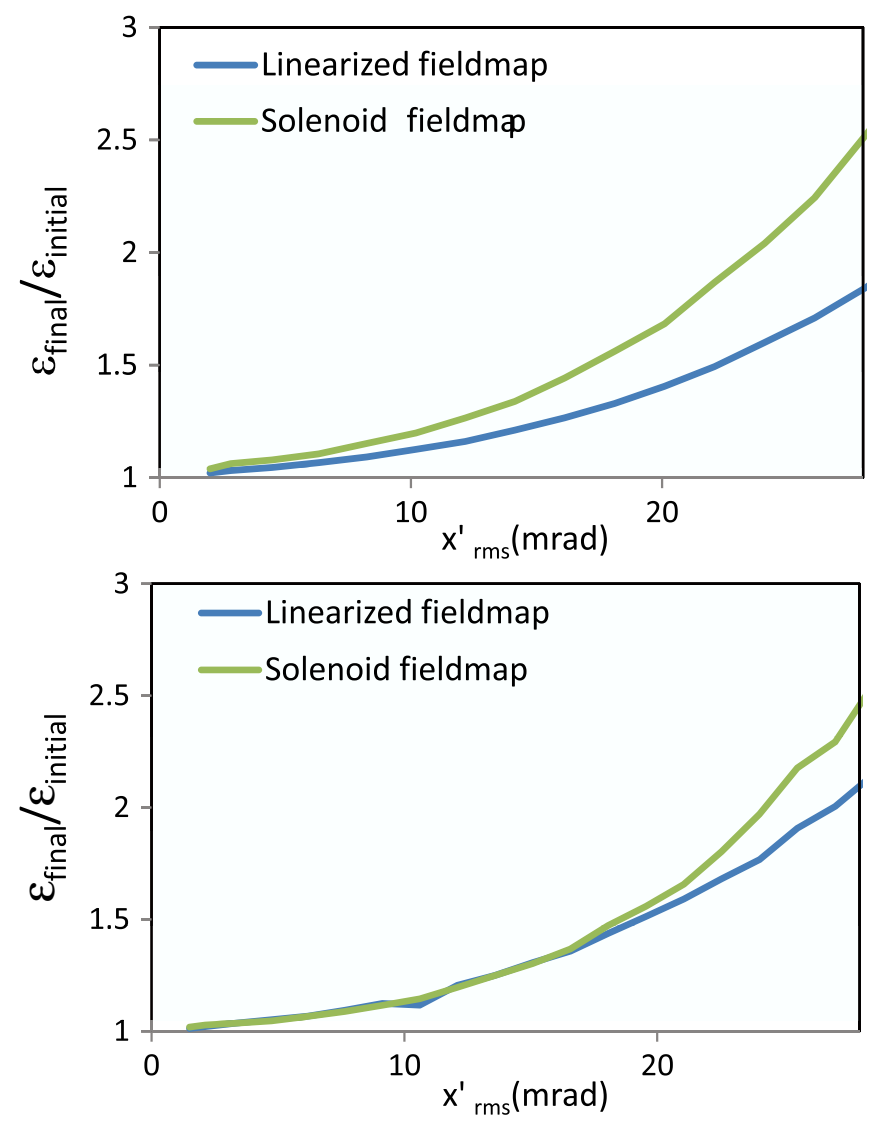

FIG. 8. (Color) Increase of emittance in solenoidal channels, $B=0.6$ T. Top: initial emittance $8.0 \pi \mathrm{mm}$ mrad. Bottom: initial emittance $4.5 \pi \mathrm{mm}$ mrad.

\section{Solenoids}

We have used the solenoid and the beam parameters of LINAC4 LEBT to confirm the analytical prediction of Secs. IIC 1 and IIC 2. We have generated beams with varying divergences (from 1.5 to $28 \mathrm{mrad}$ ), Table $\mathrm{XV}$, when entering a solenoid field as shown in Figs. 8 and 9. In another set of simulations we have varied the value of the solenoidal field from 0.5 to 1.06 Tesla, the limit value for a lossless transport. The results are reported in Fig. 7 and the extreme cases are shown in Table XVI. From the results of the calculation it is evident that the higher the beam divergence at the entrance of a solenoid, the higher the emittance increase when exiting the solenoid. In order to measure the relative effects of input beam divergence and solenoid nonlinearity we have artificially subtracted from the field map the higher order components and passed the same beam, with varying divergence through the nominal field map and through the linearized solenoid field map. The results are shown in Figs. 8 and 9, for the solenoid complete mapped field and for the linearized mapped field, i.e. $\omega_{n}=0$ for $n=1,2,3, \ldots$ Note that in the region of para-axial regime the emittance increase is negligible when the beam has a small divergence. From the plots we can conclude as the emittance decreases at
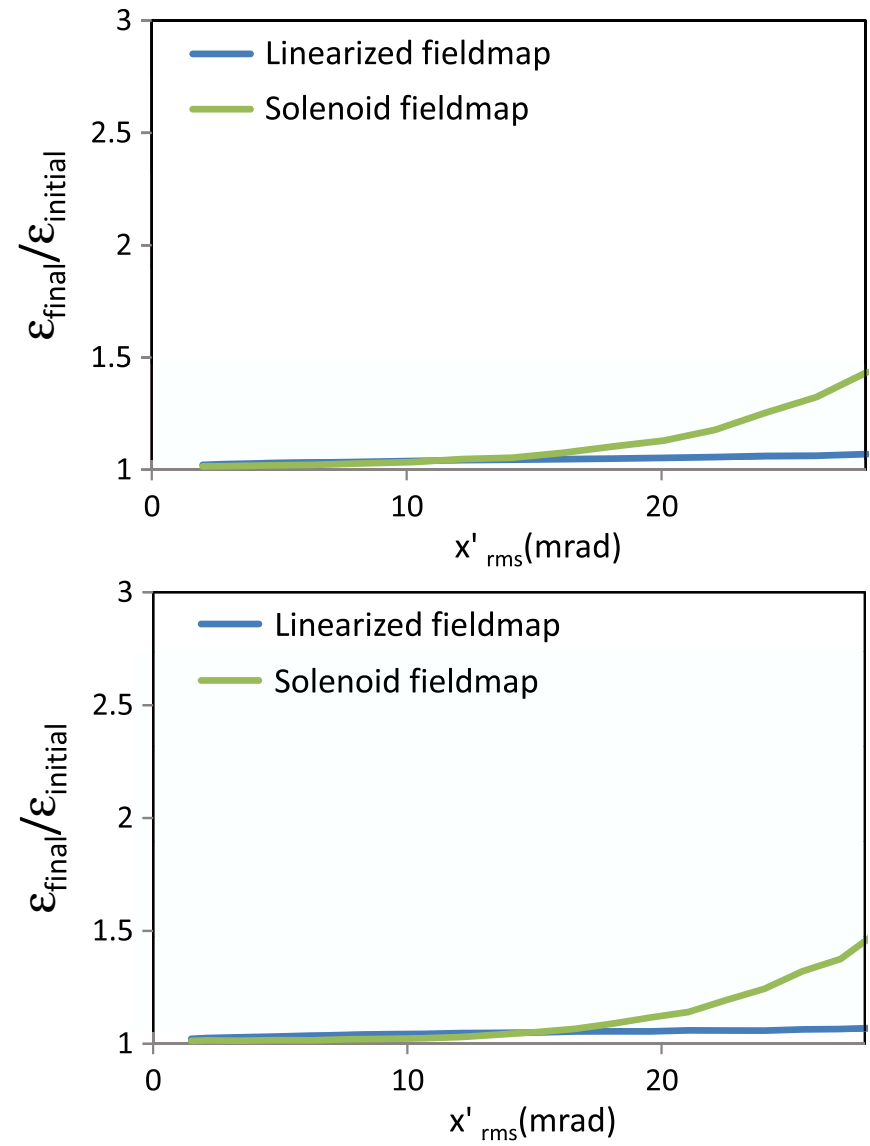

FIG. 9. (Color) Increase of emittance in solenoidal channels, $B=0.36 \mathrm{~T}$. Top: initial emittance $8.0 \pi \mathrm{mm}$ mrad. Bottom: initial emittance $4.5 \pi \mathrm{mm}$ mrad.

constant divergence (i.e. the beam size decreases), the effects of the field nonlinearities decrease and that at the limit of small emittance in a low linearized field the input beam divergence does not have any effect on the final emittance increase. On the other hand, at the very beginning of a LINAC, if one or more solenoids are used to match from the particle source to the first stage of acceleration, the designer should pay attention to minimizing the divergence of the beam when coming into a solenoid, as the emittance increase can be very severe.

\section{CONCLUSIONS}

The significant growth of the transverse emittance in $\mathrm{rf}$ cavities and solenoids motivated us to look for the optimal beam conditions to enter a cavity or a solenoid in order to limit emittance growth and beam degradation. Uniform and Gaussian beams were used to model the behavior of charged particle beams inside rf cavities and solenoids. In these studies it was shown that the emittance growth in an ideal uniform beam is less than emittance growth for Gaussian beams truncated at 3 and $5 \sigma$.

It is understood that in $\mathrm{rf}$ cavities, irrespective of the beam distribution, emittance growth and halo generation in 
beams with smaller radii are smaller while the current density is below the space-charge limit, in our case $1.4 \times$ $10^{6} \mathrm{~A} / \mathrm{m}^{2}$, and increases linearly with the fourth power of radius, as a result of slight filamentation in transverse plane. For nonparallel diverging beams emittance increase is proportional to the fourth power of the ratio of average rms beam size to initial rms beam size. Investigation of the effect of vertical and horizontal planes on each other showed that they behave independently under the rf cavity field effect and can be treated separately, excluding the effect of space charge.

An observation is made, important for the design of the LEBT solenoid. While for para-axial beams the emittance increase in a linearized field of low magnitude is negligible, it becomes important for a diverging beam ( $\sim 40 \mathrm{mrad}$ ), reaching 5 times the corresponding value for parallel beam. This result does not depend on the field strength, or the entrance beam size.

[1] L. Groening, W. Barth, W. Bayer, G. Clemente, L. Dahl, P. Forck, P. Gerhard, I. Hofmann, D. Jeon, G. Riehl, S. Yaramyshev, and D. Uriot, Phys. Rev. ST Accel. Beams 11, 094201 (2008).

[2] R. Hollinger, W. Barth, L. Dahl, M. Galonska, R. Gobin, L. Groening, P. A. Leroy, O. Meusel, and P. Spaedtke, in Proceedings of the Linear Accelerator Conference, Knoxville, Tennessee, 2006.

[3] M. Galonska, R. Gobin, R. Hollinger, and P. Spaedtke, in Ref. [2].

[4] M. Reiser, J. Appl. Phys. 70, 1919 (1991).

[5] J. Struckmeier, J. Klabunde, and M. Reiser, Part. Accel. 15, 47 (1984).

[6] F. Gerigk and M. Vretenar, CERN Report No. CERN-AB2006-084 ABP-RF, 2006.

[7] F. Gerigk, CERN Report No. CERN-2006-006, 2006.
[8] J. W. G. Thomason, H. Klein, and J. Peters, in Proceedings of 20th ICFA Advanced Beam Dynamics Workshop on High Intensity and High Brightness Hadron Beams, Batavia, Illinois, 2002, AIP Conf. Proc. No. 642 (AIP, New York, 2003), pp. 293-295.

[9] P.-Y. Beauvais, in Proceedings of the 9th European Particle Accelerator Conference, Lucerne, 2004 (EPSAG, Lucerne, 2004), p. 1273.

[10] A. M. Lombardi et al., in Proceedings of 39th ICFA Advanced Beam Dynamics Workshop on High Intensity High Brightness Hadron Beams (HB2006), Tsukuba, Japan, 2006 (CERN Report No. CERN-AB-2007-001, 2007).

[11] C. K. Allen and T. P. Wangler, Phys. Rev. ST Accel. Beams 5, 124202 (2002).

[12] P. M. Lapostolle and A. S. Septier, Linear Accelerators (North-Holland, Amsterdam, 1970).

[13] J.D. Jackson, Classical Electrodynamics (Wiley, New York, 2001), 3rd ed.

[14] M. Reiser, Theory and Design of Charged Particle Beams (Wiley, New York, 1994).

[15] D. A. Edwards and L. C. Teng, IEEE Trans. Nucl. Sci. 20, 885 (1973).

[16] G. Franchetti, Phys. Rev. ST Accel. Beams 4, 074001 (2001).

[17] J. B. Rosenzweig, Fundamentals of Beam Physics (Oxford University Press, Oxford, 2003).

[18] A. Perrin, J.-F. Amand, and T. Muetze, Travel User Manual.

[19] R. Duperrier, N. Pichoff, and D. Uriot, ICCS Conference 2002 on CEA Saclay Codes Review, Amsterdam.

[20] A. Millich and M. Vretenar, CERN Report No. PS-RFNOTE-2002-028, 2002.

[21] G. Rossat and M. Weiss, CERN Report No. PS-LI-Note85-1, 1985.

[22] K. Batchelor, Y. Kamiya, S. Okumura, H. Matsumoto, and I. Sato, Report No. KEK-ACCELERATOR-79-2, 1979. 